\title{
Autonomous Assembly of Modular Structures in Space and on Extraterrestrial Locations
}

\author{
Dean C. Alhorn \\ National Aeronautics and Space Administration (NASA), \\ Marshall Space Flight Center, ED17, Huntsville, AL, 35812 \\ 256-544-9093; dean.alhorn@nasa.gov
}

\begin{abstract}
The new U.S. National Vision for Space Exploration requires many new enabling technologies to accomplish the goals of space commercialization and returning humans to the moon and extraterrestrial environments. Traditionally, flight elements are complete sub-systems requiring humans to complete the integration and assembly. These bulky structures also require the use of heavy launch vehicles to send the units to a desired location. This philosophy necessitates a high degree of safety, numerous space walks at a significant cost. Future space mission costs must be reduced and safety increased to reasonably achieve exploration goals. One proposed concept is the autonomous assembly of space structures. This concept is an affordable, reliable solution to in-space and extraterrestrial assembly. Assembly is autonomously performed when two components join after determining that specifications are correct. Local sensors continue monitor joint integrity post assembly, which is critical for safety and structural reliability. Achieving this concept requires a change in space structure design philosophy and the development of innovative technologies to perform autonomous assembly. Assembly of large space structures will require significant numbers of integrity sensors. Thus simple, low-cost sensors are integral to the success of this concept. This paper addresses these issues and proposes a novel concept for assembling space structures autonomously. Core technologies required to achieve in space assembly are presented. These core technologies are critical to the goal of utilizing space in a cost efficient and safe manner. Additionally, these novel technologies can be applied to other systems both on earth and extraterrestrial environments.
\end{abstract}

\section{VISION AND BRIEF HISTORY OF SPACE ASSEMBLY}

Since the beginning of the space age, man has dreamed of living and working in space to discover what is out there and how do I interact with that which is and is not out there. The quest began with the launch of the Russian Sputnik satellite in 1957, continues today with the International Space Station (ISS) and the dreams go on with the vision for future space exploration as defined in the President's vision. Among the goals was to complete the ISS, send a robotic orbiter and Lander to the Moon; sending a human expedition to the Moon and conducting robotic missions to mars in preparation for a future human expedition. (Aldridge et al, 2004)

To understand this vision first requires a brief look back into the past to determine what have been our successes and failures so we can build on the success and avoid repeating the failures. For the first 10 or so years of the space age, the Americans and Russians competed to see who could top the other, and in 1969, the culmination of the race was the landing and exploration of Neil Armstrong and Buzz Aldrin on the surface of the Moon. Although the Russians launched the first space station in 1971, the next major successful event in the space race was the launch and operation of America's first space station, Skylab. Skylab would be considered the first attempt to perform assembly of components in a space environment. While the Americans focused the next 10 years on the development and construction of the Shuttle, the Russians countered with the launch and construction of the MIR space station. This event was a significant advance in space construction history. In the development of the Hubble Space telescope, it was determined that the Shuttle would service this observatory and in 1993 the first service mission, STS-31, was conducted to repair problems with the telescope. While the MIR space station continued to be operated the last and most significant construction effort in space began in 1998 and is continuing today: construction of the International Space Station (ISS). 
The ISS is considered the most ambitious construction project ever. At the date of this writing, there have been 16 Space Shuttle flights and 25 Russian assembly, crew and resupply flights. There are currently 13 different major elements providing a habitable volume of 425 cubic meters. Thirty-seven states and sixteen countries are supporting the ISS in various capacities. The ISS is serviced by the Space Shutile, Russian Progress units and soon to be serviced by the European Space Agency's (ESA) Automated Transfer Vehicle (ATV). To date, fifty-two (52) Extravehicular Activities (EVA's) have been performed so far with a total EVA time of approximately 322 hours. A total of 37 space shuttle missions are scheduled to assemble, outfit and begin the research on the ISS. This will require about 160 spacewalks totaling 960-clock hours (Petty, 2004). Figure 1 is a photograph of an astronaut during an EVA session during the STS-110 mission to the ISS. (Petty, 2004)

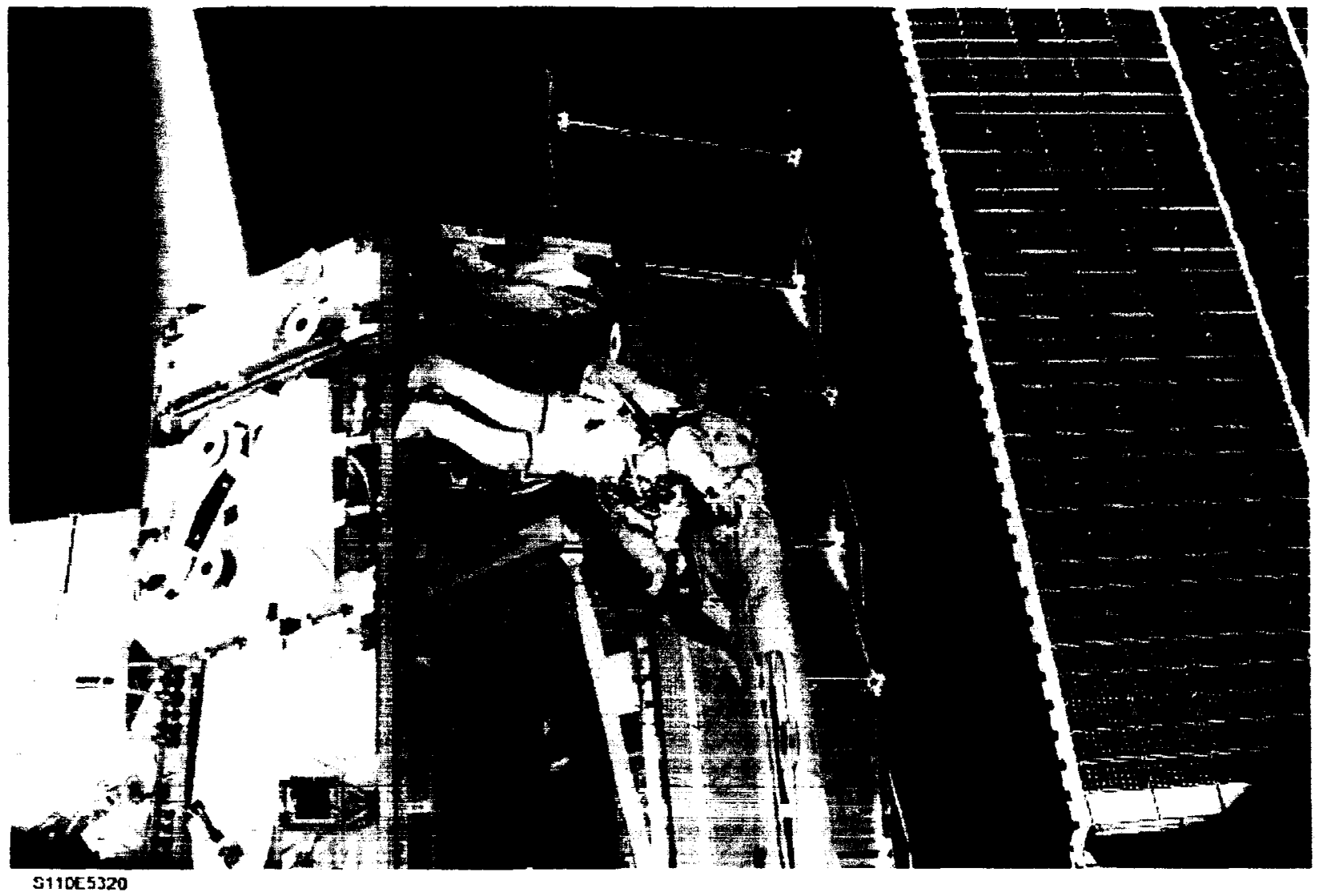

FIGURE 1. EVA Session during STS-110.

All this complexity and ambition does not come cheap. In 1998 the estimate for the total U.S. costs for constructing, assembling and maintaining the International Space Station was to exceed \$95Billion. (McCain and Frist, 1998) Each Space Shuttle launch alone costs more than \$400Million (Lindsey, 2001). According to the ISS Management and Cost Evaluation Task force report, the cost of the ISS space station in 2001 had exceeded \$139Billion. (Young, et al., 2001) It is important to note that these estimates do not include the amount that other International Space Station partners have committed to the effort. Therefore, it is surmised that the ISS project's costs will be a significant hurdle for other major visionary projects to begin. If new visionary projects have the estimated costs comparable to the ISS estimates, the new vision will not be able to generate enough interest to justify the return on investment. 


\section{CURRENT CONSTRUCTION PHILOSOPHY}

The MIR and the ISS are two prime examples of the current space assembly philosophy: Build the module as complete as possible on earth and then launch the unit to space. Once in space, the new element is connected to the existing structure with human construction oversight and/or hands on assembly via space walks. This approach to construction in space results in major elements that are heavy, bulky and must still be assembled by a human presence in orbit. Aside from the costs of designing and manufacturing these large entities, the launch costs and interface requirements are significant. Except for the Russian hardware that was launched singularly on a rocket, all other major components have to "fit" the size requirements of the space shuttle. The shuttle cargo bay can accept elements that are no longer than $18.28 \mathrm{~m}$ long by $4.57 \mathrm{~m}$ in diameter. In addition, these elements cannot be greater than $24,990 \mathrm{~kg}$ in mass (Braeunig, 1997). These requirements place significant constraints on the design and construction of major elements.

One of the proposed major ISS elements, which was a departure from this philosophy, was the TransHab module. The TransHab concept was a habitation module for the ISS. It was an inflatable living quarters as opposed to the conventional space cylinder design approach. This concept was a unique hybrid structure that combines the packaging and mass efficiencies of an inflatable structure with the load bearing advantages of a hard structure (Petty, 2004). The departure from a traditional design to this unconventional TransHab design is an evolution in the philosophy of packaging efficiency. This concept may eventually be utilized on some future mission.

\section{A NEW APPROACH}

A new way of achieving the goals for the vision of space utilization, exploration and habitation of extraterrestrial environments is to rethink the concept of assembly. Traditionally, when assembling a structure on earth, construction materials are brought to the worksite and the materials are either assembled as is, or are modified onsite to fit a particular constraint. This concept could be achieved in a modified form for construction/assembly in orbit. It is recognized that certain assembly methods that are traditionally used for joining components on earth are not feasible for physical or safety reasons. Therefore a new approach to assembly needs to be developed to achieve construction in a harsh environment with little or no direct human supervision. It is possible that direct supervision for orbiting payloads could be remotely or tele-operated by a human oversight astronaut in a safe environment; ISS, space shuttle or possibly on earth. Now, this approach would seem to be only a concept if it did not have sufficient details to make it viable.

\section{Novel Automated Assembly Method}

The first novel concept that needs to be addressed is the core idea of assembly. Assembly is defined as the putting together of manufactured parts to make a completed product. These parts would be the subcomponents of the modules or space frame trusses. A prime question needs to be addressed: Why is this different than the traditional approach? Answer: The components can be on the order of I beams and structural plates instead of shell modules and preassembled trusses. The subcomponents would be lifted into orbit or delivered to the lunar or surface by a iraditional launch. At the construction site, the components could then be assembled with various methods: By an Astronaut on an EVA mission, a free flying robotic tug, a tele-operated robotic arm or via a Robotic astronaut, i.e. Robonaut (Culbert, 2004). Figure 2 demonstrates the possibilities of this autonomous assembly concept for these types of constructions. With this concept, construction can be performed at various levels: the structural beam level, at a higher integrated level, an integrated structural component panel or at a truss segment level.

Automated and tele-operated systems can position objects to within a few centimeters of each other. A number of sensors can be used for the initial approach and alignment of the objects to be assembled. This direct feedback to the assembler, as to the location between the two objects, decreases the complexity of the system providing assembly. Thus the robotic tug, tele-operated robotic arm or robonaut would not need precision guidance to assemble two components together. This reduction in complexity lowers cost and increases the chance for mission success. 


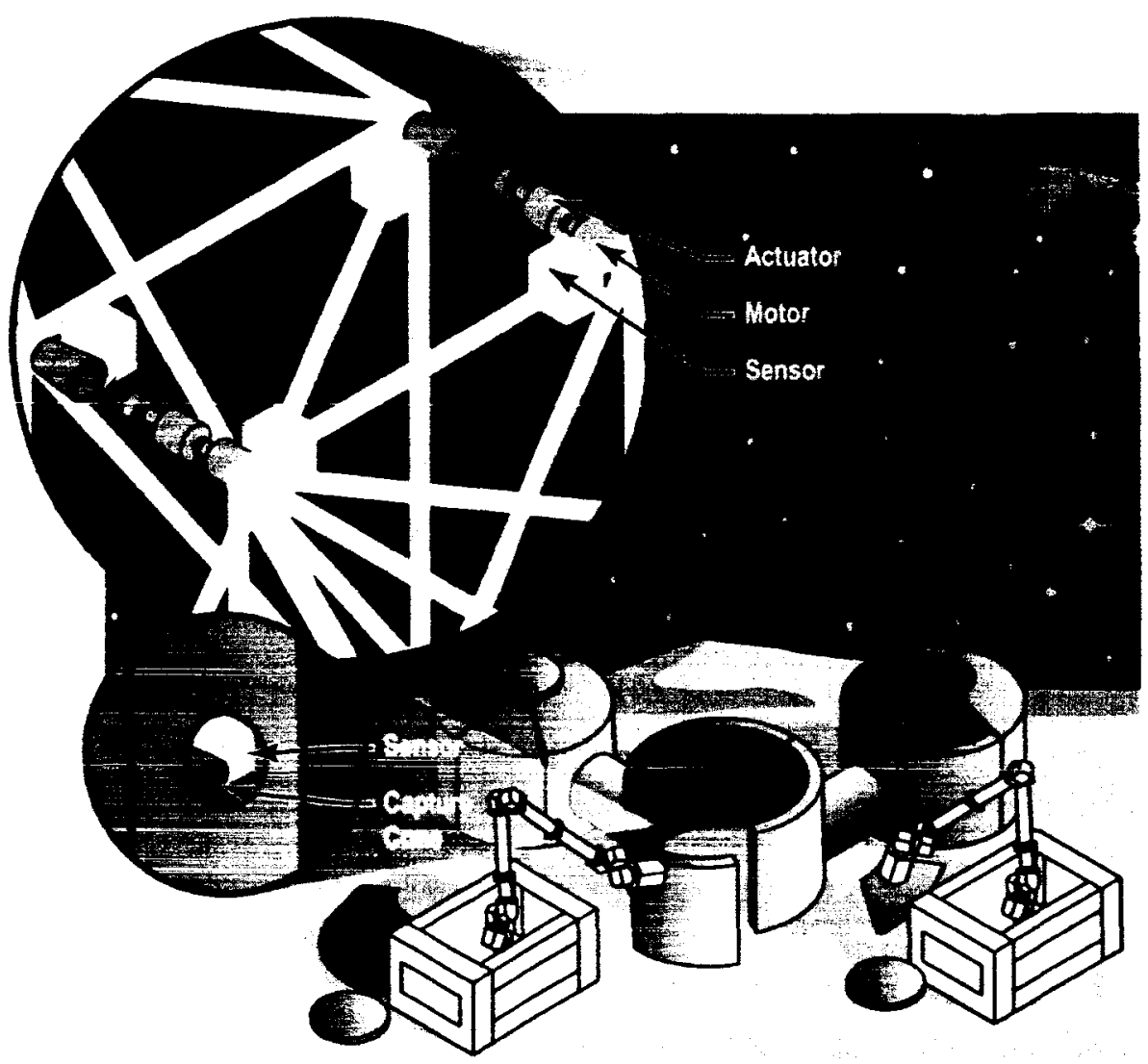

FIGURE 2. Autonomous Assembly Conceptual Application.

Referring to the enlarged detail in Figure 2, the key component in these concepts and demonstrations is the modular actuator/sensor combination used to perform the joining operations between adjacent structural components. Once the joint is assembled, the same sensor package is used to monitor the joint for structural integrity. This modular design can be used in various configurations to perform multiple joining operations. The complexity of the core unit is reduced from current electromechanical configurations, which reduces the overall complexity of assembly systems. Thus, the actuator/sensor units can be manufactured at a lower cost due to the simple design and the economics of scale for the number of units required for a fully developed system.

Assembly is autonomously performed when two components containing onboard electronics join after recognizing that the joint is appropriate and in the precise position and orientation required for assembly. The joining mechanism only activates when the specifications are correct and in a nominal range. After assembly, the sensors and electronics monitor the integrity of the joint for feedback to a master controller. In the event that a pressurized vessel is constructed, other means of securing the joint can then be performed. These could include but are not limited to: Welding, adhesive seal strips or bladder type interfaces between components.

\section{System of Connected Elements}

The avionics for this autonomous assembly system is divided into two sections: the master controller and the individual structure element avionics. MSFC has several patents $(6,254,035 ; 5,490,075 ; 5,109,345$ and others), new patent applications, and invention disclosures, which are directly related to MSFC's current research in automated rendezvous and docking. The master controller can be incorporated into a larger data system as a node and can control the mating of structure elements and monitor joint integrity. Each structural element's avionics contains the hardware required to communicate between two adjacent elements and also includes sensor signal conditioning, actuator drive, and the ability to uniquely identify each element. Element identification also includes the ability to retrieve any settings or parameters unique to that element that affects the coupling operation. 
Figure 3 shows a block diagram of the components in a fully developed system. This system contains a gap sensor technology, labeled GAP, which measures the separation between structures. The item labeled X Y is the Single Coil Absolute Position Sensor (SCAPS) device in a two-axis configuration that measures coplanar motion. These two units determine and maintain the alignment of the structural interface and joint integrity post assembly.

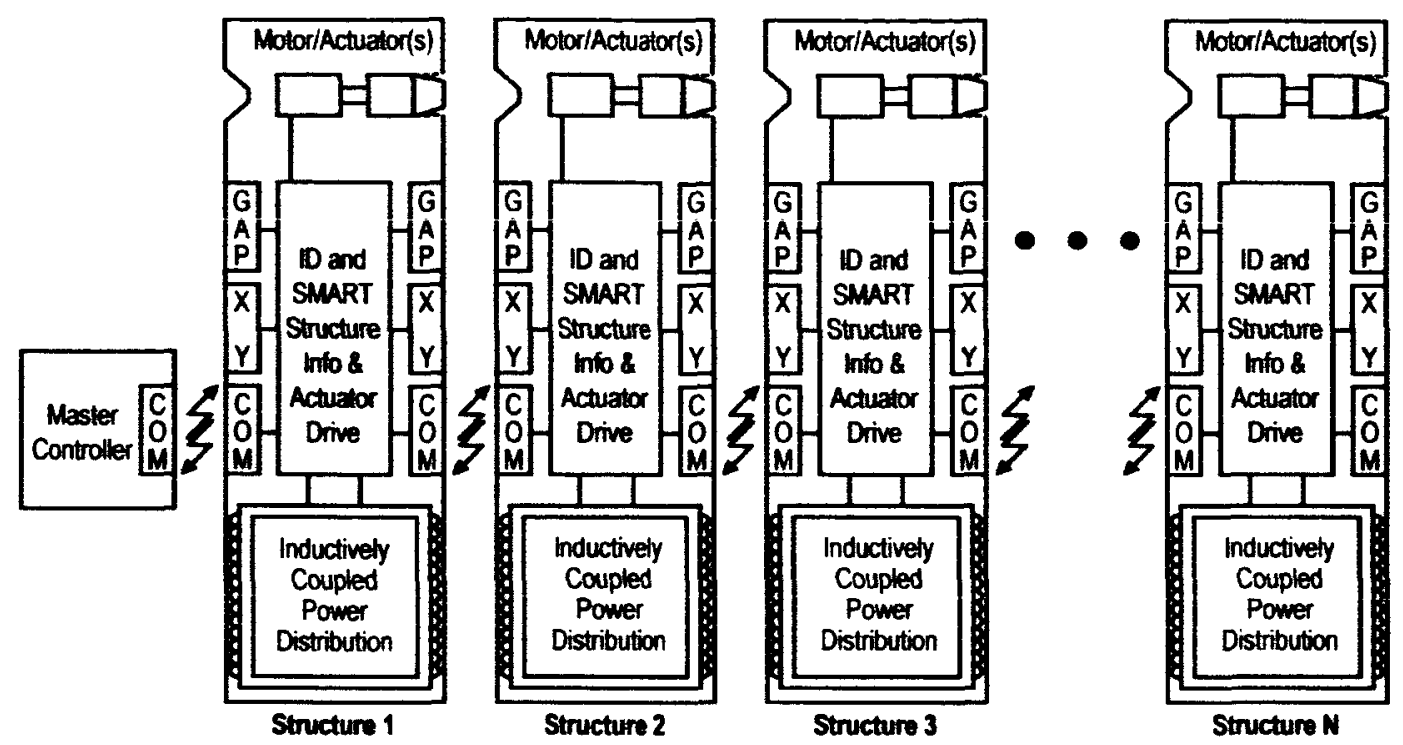

FIGURE 3. Autonomous Assembly System Block Diagram.

The item labeled COM is a close-range communication unit between adjacent structures. The Inductively Coupled Power Distribution item provides the capability to distribute power between adjacent structures by inductive coupling. The ID and SMART Structure Info and Actuator Drive contains the unique structural identification and alignment information, has the ability to receive information from the sensors, and activates the mechanism drive. If externally commanded, this device also can override internal specifications.

The Motor/Actuator component is the mechanism(s) that structurally joins adjacent components together. The Master Controller provides overriding control and insight into the mating and structural interface integrity. All electronics/sensors and mechanisms are collocated within their corresponding structure and are modularized and miniaturized.

A full system has a specific number of $\mathrm{N}$ structural elements. Each structural element contains actuator mechanisms, sensor suite, and associated control and drive electronics. The motor/actuator mechanisms align and structurally join the two adjacent elements. The sensor package provides feedback to the assembly robot/controller as to the state of the assembly process, such as position location information, health status, and joint state. As each element is added to the overall structure, the integrity of each joint is monitored by the central master controller using each element's own integrity sensor.

Individual elements are able to communicate with the master controller over the distributed network of elements. To communicate with element $\mathrm{N}$, the controller selects the ID for that element and communicates through the network to the particular structural element to determine its health or joint integrity. In a similar manner, power is transferred between elements by inductive coupling to element $\mathrm{N}$ over a distributed power network. If electrical power requirements are greater than that which can be reasonably transmitted via inductive coupling, automated connectors can be incorporated into the assembly mechanism or at another location. Initially, the assembler would supply power for each new element and the base element would receive power from a base fixed source.

The key for integrating these units is the ability to distinguish one unit from another. Each unit has a unique identification (ID) that recognizes its counterpart's ID on the adjacent structural member. If these IDs do not match, assembly is prevented. The assembly process is also prohibited, if the new structural element is not in a correct orientation. The capability is available to override the predefined IDs with a manual ID by Earth-or space-based construction monitoring personnel. 


\section{Core Sensor Technologies}

The core of this technology is a sensor suite that can monitor minute, as well as larger motions. It is redundant and reliable, miniature enough for multiple units opcrating simultaneously, and inexpensive for use in a wide range of applications. A similar application of the sensor technology is currently used in the Glovebox Integrated Microgravity Isolation Technology (g-LIMIT) flight project. The sensor concept is also being integrated into an Army missile development. Table 1 presents detailed information about repeatability and provides accuracies and errors for the sensor as tested. The relative size of a prototype sensor is shown in Figure 4. Figure 5 shows the basic internal components of these sensors.

TABLE 1. Core Sensor Capabilities.

\begin{tabular}{|c|c|c|c|}
\hline \multicolumn{2}{|c|}{ RUN-RUN REPEATABILITY } & \multicolumn{2}{c|}{ ACCURACY VERSUS REFERENCE LINE } \\
\hline Correlation (1= perfect) & 0.9999999 & Standard deviation & 0.67 microns \\
Maximum Error & 1.72 microns & Maximum Error & 3.7 microns \\
Average Error & 0.59 microns & Average Error & 0.86 microns \\
\hline
\end{tabular}

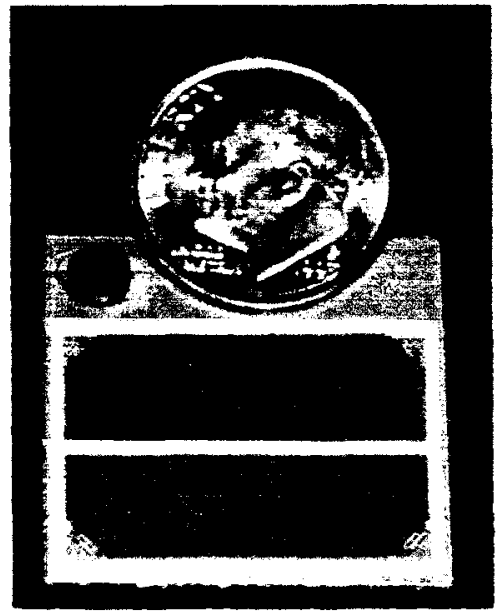

FIGURE 4. Sensor Size Comparison.
MSFC has 4 patents and 4 new technology disclosures directly related to this innovative sensor technology.
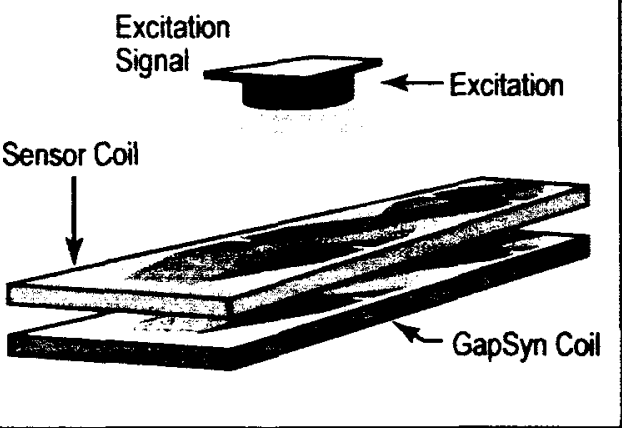

FIGURE 5. Sensor Internal Components.

Both the SCAPS and GapSyn technologies act in essentially the same manner. Referring to Figure 5, the SCAPS technology functions similarly to the reaction of a piece of metal between two magnets. If the metal is located precisely between the two magnets, it is equally attracted to both magnets and therefore the net force on the metal is zero. As the piece of metal is moved toward one magnet or the other, the forces are unbalanced. Similarly, as the SCAPS sensor excitation coil is moved away from the sensor center or null point, the net sensor output signal increases. The sensors' capabilities shown in Table 2 are enabling factors for this concept. These capabilities are: (1) measurement capacity, (2) manufacturability, (3) ability to conform to the shape and size required by a particular structure, (4) low cost due to manufacturability and the number of sensors required, (5) reliability, and (6) redundancy.

The manufacturing of this sensor technology is very inexpensive. These sensors can be made with small wire-wound inductors, printed wiring traces on Kapton, printed wiring traces on fiberglass (or other standard printed wiring boards (PWB) materials), or micro-machined in silicon, ceramic, or other appropriate material. Two sensor coils can be oriented to produce an orthogonal X-Y (two-dimensional) position sensor. Redundancy is added to the SCAPS by simply adding additional windings.

The reliability of these devices is excellent for three reasons: (1) They are non-contact devices, (2) redundancy is easily added, and (3) the materials (wire/wiring traces with a substrate of nonmetallic material) for the devices are very simple and robust. The sensor can also be embedded directly into the structural material for increased reliability in a harsh space environment. For example, the sensor could be inserted into a composite material lattice. 
TABLE 2. Sensor Technologies and Capabilities.

\begin{tabular}{|c|c|c|}
\hline Technology & Description and Capabilities & $\begin{array}{l}\text { Technology } \\
\text { Readiness } \\
\text { Level } \\
\text { (TRL) }\end{array}$ \\
\hline $\begin{array}{l}\text { Single-Coil Absolute } \\
\text { Position Sensor } \\
\text { (SCAPS), } \\
\text { MFS-32213-1 }\end{array}$ & $\begin{array}{l}\text { - Non-contact absolute linear position sensor } \\
\text { - Two active components: a sensor coil and an excitation coil } \\
\text { - Scaled up or down for various applications } \\
\text { - Linear measurements with average error less than } 1 \text { micrometer }\end{array}$ & 4 \\
\hline $\begin{array}{l}\text { Inductive Gap Sensor } \\
\text { (GapSyn), } \\
\text { MFS-32218-1 }\end{array}$ & $\begin{array}{l}\text { - Non-contact, measures the distance between two devices with } \\
\text { an anticipated operating range of } 5 \mathrm{~cm} \text { with a } 1 \text { micrometer } \\
\text { accuracy } \\
\text { - Two active components: a sensor coil and an excitation coil } \\
\text { - GapSyn sensor coil oriented on top of the SCAPS sensor coil } \\
\text { provides three-dimensional position measurements }\end{array}$ & 3 \\
\hline $\begin{array}{l}\text { Short Range Antenna } \\
\text { (SRA)/Close-Proximity } \\
\text { Transmitter and } \\
\text { Receiver, } \\
\text { MFS-32228-1 }\end{array}$ & $\begin{array}{l}\text { - Non-contact SRA/Close-proximity Transmitter/Receiver } \\
\text { - Anticipated communication rates }>1 \mathrm{Mbytes} / \mathrm{sec} \text { which are } \\
\text { comparable to the Institute of Electronic Electrical Engineers } \\
\text { (IEEE) standard } 802.11 \mathrm{~b} \\
\text { - Can be manufactured identical to the GapSyn technology but } \\
\text { with the different functions electronically multiplexed; allows } \\
\text { the same sensor to measure gaps between two devices and to } \\
\text { communicate (transmit/receive) data }\end{array}$ & 3 \\
\hline $\begin{array}{l}\text { Absolute Limit Sensor } \\
\text { (ALS), MFS-32192-1 }\end{array}$ & $\begin{array}{l}\text { - Acts as a non-contact limit switch, much like a mechanical } \\
\text { contact limit switch } \\
\text { - Measures absolute position over a } 5 \text {-mm range, with the same } \\
\text { precision as the SCAPS sensor technology }\end{array}$ & 4 \\
\hline
\end{tabular}

\section{Core Mechanism Technologies}

Mechanisms used to provide a latching capability are used in many applications. The only modifications to existing components would be for the extreme environments of space and for specific assembly requirements. These mechanisms would have the capability to be released and reactivated for testing, repair, rework or servicing purposes. The specific latch design and holding requirements would define the type of mechanism required. These could include but are not limited to: Motor/Screw drives, docking, end-effector or latching type mechanisms.

\section{ADVANTAGES AND DISADVANTAGES}

The prime advantage of autonomously assembling structures is the safety of the human oversight. It is envisioned that this concept can be remotely and autonomously performed without the need for onsite human supervision. Another advantage is the costs for launching the structure into space and transfer to its eventual destination. Fewer launches and EVA missions are required since components can be packed more efficiently. If the Robonaut concept becomes a viable resource, then human EVA missions will decline. Another advantage to this concept is modularity. If one component needs to be replaced, then the ability to insert a new component in the old location is possible. Demonstration of this concept can be achieved in a low-cost, low-risk endeavor, which would provide capabilities for a broad range of assembly needs for constructing future space facilities. The simplicity of the core technology provides a key component in the development of the complex System of Systems for space exploration. 
The main disadvantage of this concept is that it is just a concept. Implementation of the concept into a demonstration unit would provide a core technology for future space construction. Another perceived disadvantage of the concept is the ability to transfer power inductively. This disadvantage is overcome by conventional means of connectors automatically mating during the assembly process or post assembiy when the facility is habitable. Another disadvantage is the power source for lifetime monitoring. The power requirements for the monitoring electronics is low and could be inductively coupled or generated locally by solar cells.

\section{CONCLUSIONS}

Implementation of this concept is an important step in the progress of constructing future space facilities. The method by which this concept is based upon is straightforward and can be easily and inexpensively applied and scaled as appropriate. Several new cutting-edge technologies have been introduced which combine to promise relevant appropriate guidance and confirmation monitoring for automating space connections. The ability to sense, guide, and confirm successful mating is universally important for automated, human-assembled and deployed space facilities. The autonomous assembly concept as described is comprehensive in addressing a complete mechatronic capability for connection from actuation and nodal identification to status monitoring and communication of data during and after the assembly process.

The innovative absolute, non-contact sensor technology described herein has multiple capabilities to measure gap position, provide single- or dual-axis position measurement, act as a limit switch, and operate as a short-range antenna for communication. Using a single sensor suite reduces complexity, which provides increased reliability, since fewer components are required. After assembly, the sensor suite can monitor the seal integrity or relative displacement between structural components. In addition, the actual sensor component is very inexpensive, small, lightweight and can include redundant sensors collocated in the same unit.

The cost, weight, performance benefits, and capabilities of this advanced sensor position this technology as a key component for the concept of autonomous assembly of space structures. The benefits obtained from this concept are far-reaching and have significant merit. This concept and the underlying technology can also be applied to other endeavors within NASA and its global partners. In addition, the core technology can be applied to the commercial sector in such applications as high-precision alignment of electronics, optics alignment, and safety switches on highspeed rail systems.

\section{ACKNOWLEDGMENTS}

The author would like to acknowledge Steve Jones of NASA's Marshall Space Flight Center and Rene Seigh of Analytical Services Inc. (ASI) for their contribution in the original conceptual development.

\section{REFERENCES}

Aldridge, E. C. (Pete), Jr, "A Journey to Inspire, Innovate, and Discover," Report of the President's Commission on Implementation of United States Space Exploration Policy, Washington, D.C. 2004, pp. 6.

Braeunig, R. A., "Rocket and Space Technology", (1997), http://www.braeunig.us/space/specs/orbiter.htm, accessed August 5, 2004.

Culbert, C., NASA Johnson Space Center, "ROBONAUT,” (2004), http://robonaut.jsc.nasa.gov/, accessed August 6, 2004.

Lindsey, L. SpaceRef.com, "Speech by Larry Lindsey Assistant to the President for Economic Policy Speech to AAAS May 3, 2001," (2001), http://www.spaceref.com/news/viewpr.html?pid=4848, accessed August 5, 2004.

McCain, J., and Frist, W. H., "International Space Station, U.S. Life-Cycle Funding Requirements," United States General Accounting Office, Report to Congressional Requesters, Washington, D.C. 1998, pp. 1-4.

Petty, J. I., NASA Human Space Flight, "Human Space Flight Image Gallery", (2004), http://spaceflight l nasa.gov/gallery/images/shuttle/sts-110/html/s1 10e5320.html, accessed August 10, 2004.

Petty, J. I., NASA Human Space Flight, "The ISS to Date (08/03/04)," (2004), http://spaceflight.nasa.gov/station/isstodate.html, accessed August 5, 2004.

Young, A. T., et al., "Report by the International Space Station (ISS) Management and Cost Evaluation (IMCE) Task Force," Report to NASA Advisory Council, Washington, D.C., 2001, pp. 17. 